\title{
marges Marges
}

revue d'art contemporain Revue d'art contemporain

16 | 2013

Art contemporain et Cultural studies

\section{De la danse contemporaine au Maghreb à une danse contemporaine maghrébine}

From Contemporary dance in the Maghreb to a Maghreb contemporary dance

\section{Mariem Guellouz}

\section{CpenEdition}

\section{Journals}

Édition électronique

URL : http://journals.openedition.org/marges/261

DOI : $10.4000 /$ marges.261

ISSN : 2416-8742

Éditeur

Presses universitaires de Vincennes

Édition imprimée

Date de publication : 15 mars 2013

Pagination : 60-72

ISBN : 978-2-84292-366-2

ISSN : $1767-7114$

Référence électronique

Mariem Guellouz, « De la danse contemporaine au Maghreb à une danse contemporaine maghrébine », Marges [En ligne], 16 | 2013, mis en ligne le 15 mars 2014, consulté le 30 avril 2019. URL : http:// journals.openedition.org/marges/261 ; DOI : 10.4000/marges.261 


\section{De la danse contemporaine au Maghreb à une danse contemporaine maghrébine}

/1 1 Nous reprenons ici la formule de Jacques Rancière, Le Partage du sensible. Esthétique et politique, Paris, La Fabrique, 2000.
La danse contemporaine se manifeste sous différentes formes, sur de multiples supports: les spectacles, les répétitions, les cours, les institutions et les textes. Elle établit des rapports divers et différentes formes de "partage du sensible/1 ». Elle est un ensemble hétérogène réel de déterminations concrètes, mêlant discours et institutions. Elle ne s'inscrit pas seulement, sur un axe généalogique, comme la forme dominante actuelle d'une discipline artistique autonome; elle entre aussi dans un rapport de réciprocité avec les institutions, les pouvoirs et les idées qui participent aujourd'hui encore de sa construction transnationale. Tour à tour les questions du corps, la morale sexuelle, les problèmes de santé, l'incidence des sports et des techniques éducatives, les territorialisations de ses recherches (la scène, la rue, les écoles), les modes et les consommations de son spectacle et ses implications possibles dans les mobilisations sociales, peuvent affecter son travail, sa représentation et ses mythes. Poser la question de sa 
présence et de son émergence dans les pays du Maghreb c'est interroger à nouveau le statut du corps, du corps dansant, dans son rapport à l'institution et à l'État.

Une danse contemporaine au Maghreb est-elle une simple importation occidentale ou doit-elle se redéfinir selon les déterminations socioculturelles de ces pays? Pour trouver son chemin vers la contemporanéité, doit-elle imposer une technique du corps venue d'ailleurs, l'adopter et l'apprivoiser ou bien réinventer ses formes afin d'écrire à son tour sa propre histoire? Sans nous attarder sur les causes des difficultés d'émergence de la danse contemporaine dans les pays du Maghreb/2, nous souhaitons montrer comment ces deux formes de danse existent, cohabitent, se séparent et se mêlent parfois. En partant de l'analyse de deux spectacles de danse, nous tenterons de mettre en évidence ces différences entre une danse contemporaine héritière d'une tradition occidentale (une danse contemporaine au Maghreb) et une autre qui émerge des pratiques culturelles régionales, les déconstruit et crée une forme nouvelle (une danse contemporaine maghrébine).

\section{Danse, Maghreb et théorie post-coloniale}

Après la fin de l'ère coloniale, et plus encore depuis une vingtaine d'années, on s'est souvent interrogé sur la part de responsabilité de l'Afrique dans son apparent « refus » du développement. Ainsi Axelle Kabou constate, non sans sarcasme, que le rapport du continent avec les arts est particulièrement ténu: « et de fait, qu’ont gagné les Africains à être les plus forts en danse ou dans le domaine du surnaturel, si ce n'est le triste privilège de s'exclure totalement de la marche du monde réel/3? ». Au-delà de son parti pris politique et social, la sociologue camerounaise touche à la question, plus générale et philosophique, des relations évidentes entre esthétique et politique, création et pouvoir.

Sans opposer les pratiques artistiques aux besoins économiques et aux enjeux politiques, nous préférons penser la danse en termes d'agencements ou de carrefours entre les univers artistiques et les territorialités, normes et institutions de l'ordre social. Culture immatérielle et éphémère, elle ne participe pas directement à la croissance économique. Ni arme à feu ni bulletin de vote, elle ne saurait être de plain-pied sur le sol des luttes sociopolitiques. Mais ses codes et ses rituels ne peuvent pas rester étrangers aux processus esthétiques et subjectifs mis en œuvre par la mondialisation et, par conséquent, aux tensions qui en découlent, entre l'uniformisation planétaire et l'hétérogénéité culturelle.
/2 Nous avons développé ces questions dans un article précédent: Mariem Guellouz, « Corps dansant: Peut-on penser une danse contemporaine au Maghreb? ", dans Monia Lacheb (sld), Penser le corps au Maghreb, Paris, Karthala, Institut de recherche sur le Maghreb contemporain, 2012.

/3 Axelle Kabou, Et si l'Afrique refusait le développement?, Paris, L'Harmattan, 1991, p. 96. 
/4 Alphonse Tiérou, Si sa danse bouge, l'Afrique bougera, Paris, Maisonneuve et Larose, 2001.

/5 Jean-Pierre Warnier, La Mondialisation de la culture, Paris, La Découverte, 1999, p. 6.

/6 Nous nous référons ici à Gilles Deleuze et sa notion de « pensée cinéma »: L'Image mouvement, Cinéma 1, Paris, Minuit, 1985.

/7 Arjun Appadurai, Après le colonialisme, les conséquences culturelles de la globalisation, Paris, Payot, 1996.
Le titre même de l'ouvrage d'Alphonse Tiérou/4, Si sa danse bouge, l'Afrique bougera, veut répondre à l'affirmation d'Axelle Kabou. Nous sommes tentés ici d'en développer une paraphrase: "Si sa danse bouge, le Maghreb bougera ». Le corps dansant, tel un diapason, informe et dit quelque chose des dissonances ou harmonies qui entourent sa performance.

Les flux artistiques et culturels, au travers des médias, s'exportent de plus en plus rapidement, mais leurs mouvements et leurs succès sont inégaux. On ne peut réfléchir sur le devenir de la danse contemporaine au Maghreb sans prendre en compte le chemin inverse, la réception des danses du Maghreb en Occident. Cet accueil est plein d'équivoques. Qu'a retenu l'Occident des danses arabes à part le spectacle de danseuses peu couvertes s'offrant aux touristes voyeurs des clubs de vacances? Les danses arabes, dites « orientales » ainsi que les danses berbères, comme les danses tziganes et gitanes ont souffert de l'expansion capitalistique. Le profit et le marché les ont transformées, commercialisées. À rebours, Warnier voit bien que «la culture des eskimos ou des banlieues ouvrières françaises est étroitement localisée et n'a ni l'ambition, ni les moyens de se diffuser mondialement. [...] L'industrie fait intrusion dans les cultures-traditions, les transforme et parfois les détruit/5\%. Ainsi se profile clairement le destin des cultures «mineures». Le corps maghrébin, au bout des chaines sociales de transformation, n'échappe pas aux lois et processus de la consommation planétaire. Son être et son apparaître sont sollicités ensemble, avec ou à distance des coutumes et morales originelles.

La danse contemporaine se présente de prime abord comme une "pensée danse/6 », c'est-à-dire une façon d'être, de penser l'art et son rapport à la contemporanéité. Cette pensée est aux prises, sur un mode nécessairement conflictuel, avec les données socio-économiques et institutionnelles de la « postmodernité ». La mondialisation accélère les flux culturels en oscillant entre les deux pôles de l'homogénéisation et de l'hétérogénéisation. Ces notions, empruntées à Appadurai/7, proposent d'échapper à la stigmatisation d'une période encore post-coloniale, capitalistique et impérialiste. Elles tentent de dire que la globalisation est un processus présent et concret, ni bon ni mauvais en soi, qui transforme profondément nos subjectivités, bouleverse les pensées et les corps.

La danse contemporaine s'y trouve incluse. Occidentale, née dans les pays riches, post-industrialisés, laïques, «libérés » sexuellement, elle devra se trouver une légitimité éthique et esthétique, un discours, une corporéité nouvelle et un public prêt à l'accueillir. Les mondes d'accueil sont ici ceux du Maghreb, qui emprunte déjà depuis 
longtemps certaines tendances d'un apport étranger devenu de plus en plus familier ou intrusif.

Après la colonisation (territoriale, économique, intellectuelle, linguistique) peut-on parler encore de la culture de l'autre comme d'une culture étrangère? Dominante, elle l'a été jusqu'à la fin du millénaire, certes, mais l'étrange et l'étranger ont changé de camp: ils caractérisent maintenant le corps maghrébin tel qu'il surgit dans la représentation occidentale. Jean-Pierre Warnier décrit, non sans verve, les brassages culturels: " on danse le tango à Paris, le bikutsi camerounais à Dakar, la salsa à Los Angeles. McDo sert ses hamburgers à Pékin, et Canton sa cuisine à Soho. L'art Zen du tir à l'arc bouleverse l'âme germanique. La baguette parisienne a conquis l'Afrique de l'Ouest. À Bombay, on voit le pape en mondovision. Les Philippins pleurent les obsèques de la princesse de Galles/8... ». La mondialisation technologique est devenue un phénomène banal et quasi naturel. Les prédictions de Marshall McLuhan avec la notion de «village global » sont aujourd'hui pleinement vérifiées.

La danse contemporaine n'échappe pas à ces déterminations et à ces mouvements planétaires, elle s'est implantée dans les pays du Maghreb depuis quelques décennies et elle essaye de trouver ses parcours et ses lignes de fuite.

\section{De la danse contemporaine}

La pluralité des sens pourrait déjouer toute tentative de définir la danse contemporaine. Le qualificatif comporte l'ambiguïté sous-jacente à ce syntagme. Contemporain renvoie autant à une détermination temporelle, au sens d'" actuel ", qu'à un sème générique référant à des traits sémiques divers caractérisant la danse dans son essence et dans sa technique. S'agit-il donc de temporalité, d'histoire, ou bien d'une technique du corps, voire d'une phénoménologie? L'origine de la danse moderne est généralement associée à Isadora Duncan et sa naissance est liée aux changements économiques, sociopolitiques et historiques de la fin $\mathrm{du} 19^{\mathrm{e}}$ siècle. Cette nouvelle danse ne fait pas rupture avec le classicisme. C'est le travail de Cunningham qui introduit un véritable changement, une "rupture épistémologique », dans la pratique de la danse et les logiques du corps: «l'histoire de la danse moderne est littéralement partagée en deux par cette œuvre à la fois révolutionnaire et fondatrice: il y a l'avant et l'après Cunningham/ $\mathbf{9}$ ". Modernes, postmodernes, classiques et traditionnels se réfèrent tous à la temporalité. Si le traditionnel et le classique s'opposent au moderne et au postmoderne, il est par contre difficile de dissocier le moderne du contemporain. Maintenant toutes ces danses sont dites
/8 Jean-Pierre Warnier, op. cit, p. 3.

/9 Isabelle Ginot et Michelle Marcel, La Danse au XXe siècle, Paris, Larousse, 2007, p. 138. 
/10 Viviane Lièvre, Danses du Maghreb. D'une rive à l'autre, Paris, Karthala, 1987, p. 7.

/11 Mauss Marcel, Sociologie et anthropologie, Paris, PUF, 1950.

« contemporaines ». Qu'est-ce qui distingue alors le contemporain de la danse " contemporaine »? Quelle sera l'appellation adéquate de la danse « contemporaine » dans une cinquantaine d'années?

Celle-ci est manifestement assimilée à une attitude, un projet à la fois artistique et social, marqué par sa transversalité, une non-délimitation de son domaine et de ses objets et moyens. Ce qui est commun à ses formes polyvalentes serait justement la production des différences et des singularités, le refus de se soumettre à des règles, des normes et des techniques préétablies. Cette danse, qui ne revendique aucune généalogie, donne l'impression d'une émergence ex nihilo, d'une totale nouveauté.

La danse contemporaine repose essentiellement sur l'improvisation et la création incessante du nouveau. La rupture codique (avec le discours, la narration, le costume et toute forme de signifiance) est ici axiomatique. Les danses traditionnelles du Maghreb, en revanche, sont définies et codifiées; elles répètent avec peu de variations un modèle de formes chorégraphiques. Le corps dansant y prend la place d'un signifiant dont le signifié appartiendrait au contexte historicosocial. Ce mouvement d'abstraction est la logique où se crée la différence entre deux façons de penser le corps et l'art. Rupture esthétique, mais aussi culturelle et sociale.

\section{Les danses du Maghreb}

Les danses traditionnelles du Maghreb se caractérisent par leur multiplicité. L'ethnologue Viviane Lièvre souligne pourtant la rareté des travaux qui les concernent: «Personne ne s'étonne de trouver des ouvrages sur les danses indiennes ou balinaises, sur le buto japonais, le flamenco ou le tango argentin, facilement disponibles en librairies ou en bibliothèques. Des écrits aussi encadrés par de multiples photos sur les danses d'Afrique noire... mais sur les danses d'Afrique du Nord, on a beau chercher, il n'existe pas l'ombre d'une référence dans le vaste champ de l'édition/10. ".

Nous reprenons à notre compte la notion de «technique du corps» et voulons penser ces danses comme un objet social. Les techniques du corps, selon Marcel Mauss/11, découlent de la faculté qu'ont les hommes d'instrumentaliser leurs corps. Il s'agit d'un habitus du corps, de ses pratiques. Le corps dansant n'échappe pas à cette mémoire, au carrefour de l'individuel (la biographie), du collectif et du social. Les danses du Maghreb se dansent en solo ou en groupe de femmes, d'hommes ou mixtes. Ces danses sont exposées, elles aussi, aux processus transnationaux d'homogénéisation (ballet, conservatoire, écoles, institutions...) et d'hétérogénéisation (pratiques tribales, 
ethniques, rituels religieux). Cependant, d'autres danses, venues d'ailleurs, ont aussi trouvé leur place dans le champ artistique. Des cours de danse classique, modern jazz et contemporaine ont vite vu le jour. Des compagnies, des théâtres et salles de spectacles ouvrent leurs portes et un nouveau public émerge. Depuis quelques décennies des tentatives plus ou moins réussies pour faire exister la danse contemporaine au Maghreb se multiplient. Des chorégraphes et des danseurs tentent de donner à cette pratique sa légitimité.

Deux tendances artistiques se profilent. Une première regroupe des chorégraphes qui se situent dans le sillage des techniques corporelles occidentales, qu'ils adoptent, retravaillent et transmettent. La seconde concerne les chorégraphes de danses populaires qui, dans un travail de déconstruction, font émerger des formes nouvelles et contemporaines des techniques traditionnelles.

\section{La danse contemporaine au Maghreb}

La danse contemporaine n'a pas encore acquis ses lettres de noblesse au Maghreb. Elle reste une pratique étrangère et non tout à fait légitime. La question de la possible existence d'une danse contemporaine au Maghreb interroge le statut de l'art contemporain en général dans ces pays. Il nous faut souligner l'absence de musée d'art moderne ou d'art contemporain au Maghreb. En Tunisie, la future Maison de la Culture en abriterait un, qui reste pour l'instant à l'état de projet. À part Le Caire où se trouve le Musée d'art égyptien moderne (mathaf al-fan al masri al-hadith/12), les pays du Maghreb ne bénéficient pas, à notre connaissance, de lieux d'exposition institutionnels d'arts modernes et contemporains. Ainsi, le plasticien Naceur Ben Cheikh constate l'absence d'une tradition, d'un public: «En dehors de l'aspect didactique et doctrinaire qui se dégage de cette volonté déclarée de cultiver la curiosité du public, nous pouvons, quant à nous, constater le désintéressement à l'égard d'une activité culturelle parachutée, de la part d'une société qui ignorait jusqu'à l'existence même de l'art de la peinture en tant que pratique autonome. On peut même avancer que si des artistes continuent à produire, c'est encore grâce à l'encouragement du secteur étatique et semi étatique de l'activité économique et des achats de l'État. Ce qui, dans la plupart des cas, transforme la peinture en discours idéologique/13 ».

La faille institutionnelle concerne l'art contemporain dans son ensemble, la danse y compris. Plusieurs tentatives, entre réussite et échec, ont contribué à la faire connaître et à lui donner une légitimité institutionnelle et sociale. Des écoles, des festivals, des rencontres internationales témoignent de cette ambition. Cependant, trois facteurs
/12 Sylvia Naef décrit ce musée comme " un concept muséographique peu satisfaisant: œuvres mal ou peu étiquetées, un personnel non qualifié, pas de matériel d'information, exception faite d'un catalogue qui n'indique même pas les dates des œuvres ». Sylvia Naef, « Entre mondialisation du champ artistique et recherche identitaire. Les arts plastiques contemporains dans la Méditerranée orientale », dans Jocelyn Dakhlia (sld), Créations artistiques contemporaines en pays d'islam. Des arts en tension, Paris, Kimé, 2006, p. 85, p. 71-97.

/13 Naceur Ben Cheikh, Peindre à Tunis. Pratique artistique maghrébine et histoire, Paris, L'Harmattan, 2006, p. 156. 
/14 La récente affaire de la projection du film de Marjane Satrapi Persépolis en Tunisie est un exemple accablant. Une représentation imagée (bande dessinée) de dieu dans le film a suscité la colère de quelques personnes et a été suivie par des attaques verbales et corporelles à l'encontre des membres de la chaine de télévision en question.

/15 Sylvia Naef, op. cit., p. 71. majeurs, que nous ne développerons pas ici, ont limité l'expansion de cette pratique dans les pays du Maghreb : l'institution absente, le public non avisé et le statut de l'artiste.

Une autre difficulté échappe aux déterminations socio-économiques et touche à la question du corps dansant lui-même et du désir de danse. L'art contemporain, au Maghreb, vient bouleverser les multiples rapports au corps et à ses représentations. Dans des pays où la question de l'image est encore problématique/14 et l'Islam souvent iconoclaste, l'image et les représentations imagées réitèrent continuellement son statut. On comprend aisément ce qu'il en est (ou serait) du corps dansant, habillé ou nu. Pourtant, lors des cérémonies de fêtes, les Maghrébins dansent et ne montrent aucune réticence à exhiber leur corps, ses ondulations, ses frénétiques secousses et tremblements. Les cérémonies rituelles et religieuses sont aussi parfois accompagnées de danses (de groupe, de transe...).

Au Maghreb, au-delà de certains interdits religieux ou de certaines prescriptions conservatrices, les corps dansent. Le passage d'une danse ethnique ou folklorique, lestée de sa fonction sociale, à une création artistique qui présente le corps comme instrument et objet d'art nécessite une rupture éthico-esthétique. Dans des pays où l'homosexualité est considérée comme un délit majeur et est passible d'une peine de plusieurs années de prison, où la sexualité est juridiquement réglementée (adultère, sexualité hors mariage, polygamie...) l'autonomie du corps, d'un corps dansant qui échapperait à toutes ces contraintes n'est pas encore envisageable.

Pourtant plusieurs chorégraphes/danseurs tentent de résister, de lutter contre toutes les formes de préjugés ou de censure. Ainsi, en Tunisie par exemple, une explosion chorégraphique se démarque actuellement des difficultés que rencontre cette danse dans les autres pays du Maghreb. Imed Jemaa, Sondos Belhassen, Radouane EIMedeb, Malek Sebai, Nawel Skandrani, Iman Smeaoui et beaucoup d'autres sont devenus des artistes reconnus. Ils bénéficient parfois de salles de spectacle, de subventions et de festivals. Sylvia Naef raconte le processus d'historicisation de l'art moderne dans la Méditerranée orientale: « l'apparition des arts modernes, inspirés de l'art occidental date, dans l'Empire ottoman (dont la majorité des pays arabes faisaient partie), du $19^{\mathrm{e}}$ siècle. Au début, la rupture avec des pratiques locales, savantes ou populaires, fut volontairement totale. Il s'agissait, dans le cadre d'un processus de modernisation parfois violent, de tourner le dos aux traditions et s'approprier les sciences et les techniques de l'Occident, dont on considérait que les beauxarts faisaient partie/15 ». Le processus d'apparition et de propagation des pratiques artistiques contemporaines est donc nécessairement 
tributaire de la question coloniale. Et celle-ci comporte deux problématiques: celle de la politique globale (les rapports entre culture et mondialisation) et celle d'une micropolitique (le rapport au corps et les ruptures avec ses modes d'aliénation). Ainsi, même si la danse contemporaine en tant que pratique occidentale, post-industrielle, a trouvé des chemins possibles dans les pays du Maghreb, sa légitimité et sa persistance sont encore menacées.

\section{Vers une danse contemporaine maghrébine}

Après avoir évoqué les possibilités d'émergence d'une danse contemporaine au Maghreb nous en examinons maintenant une autre forme. Certains danseurs remontent le temps et tentent de retrouver une forme qu'ils pensent être proche du pas traditionnel. Dans un premier temps, une volonté de sauvegarde et de transmission des danses folkloriques leur semble nécessaire; puis vient un travail de réinvention. Plusieurs exemples illustrent ces explorations artistiques. À la frontière du Maghreb, on peut citer le travail de Souraya Hilal. La danseuse égyptienne a développé depuis quelques années une danse orientale contemporaine. En puisant à nouveau dans le pas traditionnel des danses égyptiennes du Haut Nil, en les dépouillant, en effaçant les différenciations sexuelles, elle a créé une forme nouvelle, non immédiatement facile à répertorier. Un point de vue critique reconsidère les costumes, apparats et mimiques de la danse arabe. Celleci se débarrasse de ses codifications, explore d'autres techniques, d'autres musiques, d'autres postures. Elle construit une nouvelle identité, moins localisée, plus abstraite de la géographie.

Nous nous attarderons sur deux autres exemples : le premier est celui de la chorégraphe tunisienne Malek Sebai et le second celui de la chorégraphe franco-algérienne Saâdia Souyah. Les deux ont des formations et des techniques différentes. La première vient du monde de l'opéra et du contemporain, la seconde de celui des danses d'Égypte et du Maghreb. Leurs recherches se recoupent mais diffèrent à plusieurs niveaux esthétiques et techniques.

\section{Malek Sebai : le choc des corps entre tradition et modernité}

Malek Sebai est une danseuse et chorégraphe tunisienne. Après une formation et un stage en danse classique à l'Opéra de Paris en 1978, elle a intégré le Conservatoire national supérieur de danse et de musique de Paris jusqu'en 1984. Elle s'installe à Tunis en 1998 où elle enseigne la danse classique et contemporaine. Elle se consacre 
à un travail de recherche chorégraphique depuis des années et tente malgré de nombreuses difficultés de faire exister cette pratique en Tunisie. Dans sa dernière création, Khira et Ruchdi, la chorégraphe explore un nouveau terrain, celui des danses tunisiennes, et un nouvel univers, celui des cabarets.

La pièce est composée pour deux danseurs et un percussionniste. Un duo met en scène la danseuse Khira Ben Oubeidallah, figure majeure de la danse traditionnelle tunisienne (telle qu'elle se pratique dans le ballet) et le danseur contemporain et marionnettiste Ruchdi Belkacmi. Le choc est double. D’abord un choc générationnel: la danseuse sexagénaire, ancienne maître de ballet dans la troupe des arts populaires de Tunisie, donne à voir un corps quasi archéologique, un corps devenu lui-même mémoire et trace de ce mouvement traditionnel. En face d'elle, un jeune danseur nous offre un corps dansant sculpté, à la fois masculin et féminin, multiple, traditionnel et contemporain. Les deux se confrontent: chacun sa formation, chacun sa technique. Le percussionniste rythme leur rencontre. Comment bouger dans un corps vieillissant? Comment trouver la présence dans un corps novice ? La danseuse se retire. Ruchdi se déshabille et dévoile presque avec arrogance ce corps jeune, musclé, fluide. II met une jupe bleue, noue l'écharpe blanche spécifique des danses tunisiennes autour du bassin (hzam) et danse. Cette danse part du mouvement twisté tunisien mais l'abandonne en cours de route. Le danseur explore tous les possibles de ce mouvement, il le décortique, l'ornemente, lui greffe d'autres gestes venus d'ailleurs ou de nulle part, le répète jusqu'à ce qu'on ne le reconnaisse plus. Le pas de la danse tunisienne s'efface peu à peu mais son ombre est présente. Ruchdi se contorsionne, se perd, nous égare. Dans sa jupe, dans son corps mince quasi androgyne, toutes les dichotomies s'abolissent: femme/homme, adulte/ enfant, traditionnel/contemporain. II ne reste que l'effet, le ressenti. Le danseur explore la danse ancestrale avec son corps d'homme tunisien, maghrébin, après la Révolution, après les années de dictature et enfin avec la victoire des islamistes aujourd'hui. Passé douloureux, futur incertain, il danse cet entre-deux, mélange les mondes et les techniques et fait ainsi émerger une forme sobre, dépouillée, puissante, contemporaine du mouvement millénaire, sous les impulsions des rythmes de la musique tunisienne (fezzani et bounaoura). Khira revient sur scène, se réimpose et impose son corps lourd, elle éclate de rire, le regarde et quelque part cède la place et consent à partir. Les deux danseurs se retrouvent à nouveau dans un duo. Ruchdi remet le pantalon et dans un mouvement et un rythme répétitifs, ils explorent tous les deux les ressentis de la transe. Le public est pris dans cette ambiance qu'il connaît comme pratique rituelle, mais ne la reconnaît 


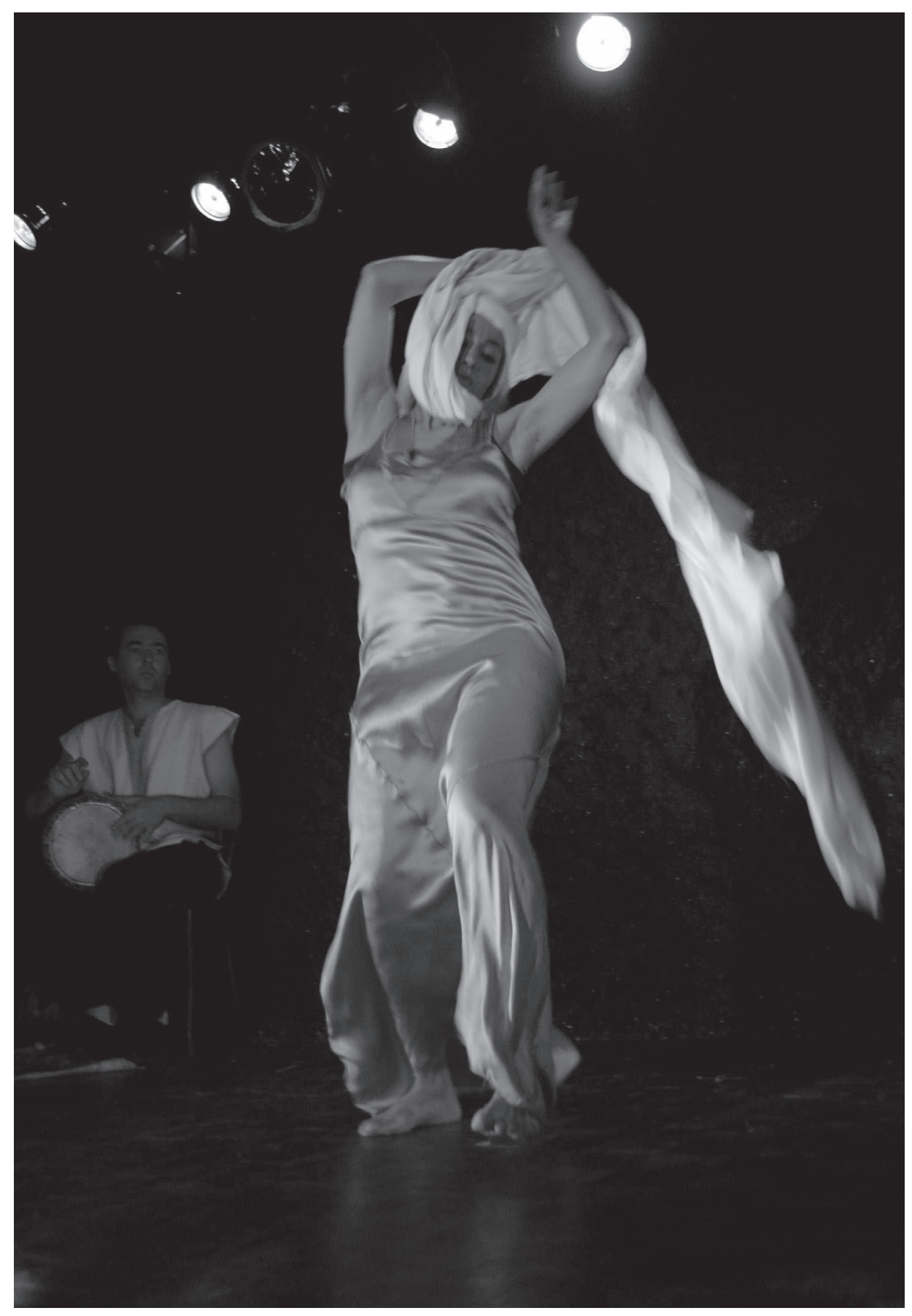


/16 Elle vient de créer récemment le Centre des danses arabes et écritures contemporaines à Paris.

/17 Safaa Monqid, "Les Marocaines et la danse: un espace d'expression ", dans J. Deniot, A. Dussuet, C. Dutheil et D. Loiseau (sld), Femmes, identités plurielles, Paris, L'Harmattan, 2002, p. 121-145. pas tout à fait. Un trouble, une gêne, gagne le spectateur. Les repères identitaires, nationaux et folkloriques sont déstabilisés, déconstruits. Une nouvelle danse, un nouveau monde naît.

\section{Saâdia Souyah : bouleverser la tradition}

La danseuse et chorégraphe franco-algérienne Saâdia Souyah est spécialisée en danses du Maghreb et d’Égypte, qu'elle enseigne en Europe. Elle élabore un travail de recherche chorégraphique autour des danses arabes et berbères. On peut tenir son travail artistique pour une recherche quasi anthropologique. Dans sa volonté de fuir les a priori et les imaginaires orientalistes elle tente de redonner à la danse maghrébine une dimension foncièrement artistique. La chorégraphe multiplie les voyages, les rencontres avec des autochtones. Les vidéos de danses au cours de cérémonies et de rituels sont pour elle un élément de travail important/16.

À partir d'une danse marocaine saharienne appelée la guedra, la chorégraphe fait émerger une forme nouvelle. La guedra est une danse marocaine de la région des Oued Noun. II s'agit de la pratique d'une tribu caractérisée par un brassage culturel entre des tribus arabes, berbères et des communautés noires. Cette danse se pratique dans des cérémonies rituelles de célébration. Selon un groupe d'ethnologues qui a rendu compte des danses des femmes du Maroc, la danse de la guedra serait « constituée d'un groupe d'hommes (plus de vingt personnes), qui s'installent à genoux, serrés les uns contre les autres [...]. Les hommes débutent la danse en battant des mains en cadence pour soutenir le rythme de la guedra, tout en bougeant leurs épaules. [...] Une danseuse se présente au milieu du cercle, soutenue par les youyous des femmes. Toute voilée, elle commence à danser, laissant ainsi les hommes deviner son corps et suscitant par-là leur curiosité et leur désir. Cela ne dure pas longtemps puisque l'un des participants ou parfois la "Khadem" (esclave) qui l'introduit au centre du cercle, lui ôte le voile, dévoilant ainsi son visage et sa longue chevelure/17 ».

Saâdia Souyah s'inspire de cette danse. Une tension double caractérise son travail: dépouillement et exagération. En effet, elle ne retient que le mouvement des mains, le jeu des doigts. Elle met de côté le caractère ludique et enjoué de la guedra au profit d'une présence grave et puissante. Elle exagère le regard, l'intensité, les secousses du corps. On passe d'un univers de cérémonie rituelle où hommes et femmes célèbrent ensemble l'amour et les saints à une forme sobre et étrange. Le regard de Saâdia Souyah est théâtral, tourné vers ses propres doigts comme si elle les découvrait pour la première fois. On s'éloigne de la guedra pour s'approcher d'une technique caractéristique de la 
danse japonaise contemporaine, "le buto ", ou lenteur, secousses et regards sont essentiels. La danseuse s'émancipe de la stylistique marocaine, lui ajoute un mode extrême oriental et crée ainsi un univers nouveau, un peu fou, étrange. Le public garde tout de même quelques repères comme la musique, le voile noir, la position assise et les mouvements de mains; mais il est bousculé par le regard hanté de la danseuse qui pointe un doigt comme s'il était étranger à son corps. Une gestuelle vers l'avant du corps invente comme par surprise une nouvelle posture. Les tissus alourdissent le corps de Saâdia Souyah, le prolongent et semblent l'immobiliser. Entre buto, danse contemporaine, transe et guedra, tous les codes sont métissés. Dans ce processus de création de nouveau, la danseuse greffe dans son corps dansant des gestes étranges et étrangers. Elle échappe au public maghrébin, le déroute. Elle danse un corps de femme citadine, émancipé des langages et des repères délimités. Un corps qui est à la fois trace anthropologique et lieu de réinvention de la tradition. Un corps dansant fait de mémoire et d'oubli.

\section{Et si nous n'avions jamais été contemporains/18?}

D'une danse contemporaine qui essaye de trouver sa place et sa légitimité parmi les arts et les pratiques artistiques au Maghreb à une danse contemporaine maghrébine, il n'y a pas nécessairement de rupture. Le travail de Malek Sebai en est témoin. Celle-ci, après avoir été imprégnée par une formation classique à l'Opéra de Paris et contemporaine à New York, a contribué à l'implantation et à la transmission de cette danse en Tunisie. Dans son dernier spectacle, elle parcourt un chemin contraire. Elle revient sur la danse tunisienne qu'elle regarde du point de vue de sa formation de chorégraphe contemporaine. La danse contemporaine maghrébine semble exister dans ces explorations et ces métamorphoses des danses ethniques et folkloriques, dans les mélanges et les expérimentations des techniques venues d'ailleurs, d'ici ou de nulle part.

On peut parler de deux formes de danses contemporaines au Maghreb: l'une influencée par les techniques occidentales, s'adaptant et se soumettant aux contraintes rencontrées; l'autre s'appuyant sur une démarche de déconstruction réinventant les danses ancestrales. La danse contemporaine au Maghreb ou la danse contemporaine maghrébine, dans le sillage des récents progrès démocratiques, s'annonce sous l'impératif de la multiplicité. L'art contemporain en général et la danse contemporaine en particulier doivent réécrire leur histoire dans les pays du Maghreb, trouver leur forme propre, se mélanger, s'exporter et se déterritorialiser.
/18 Nous paraphrasons ici le titre du livre de Bruno Latour, Nous n'avons jamais été modernes, Paris, La Découverte, 1991. 
/19 Abdelkebir Khatibi, L'Art contemporain arabe, Paris, éd. Al Manar/ Institut du monde arabe, 2001, p. 125.
Khatibi se demande: «Où va le monde arabe? Sa civilisation? Qu'en sera-t-il de ses arts, en cette vitesse informatisée des images, des sons et des techno-sciences de mémorisation/19?». Cette interrogation entraîne nécessairement une réflexion sémiotique. Une civilisation du signe, du Livre, peut-elle se dégager et s'autoriser d'ellemême une sémiotique de l'image? Comment établir une intersémioticité entre le visible et le lisible? Comment donner au corps le droit de se raconter, de s'exposer, comme corps, tel qu'il est dans sa beauté et dans sa laideur, dans ses apparats et dans sa nudité? Un corps subjectivé, un corps qui m'appartient en tant que sujet, que j'arrache à l'État, aux intégrismes, à la famille, à la prière, à l'institution.

Khatibi pense à la calligraphie comme possible sortie du linéaire du livre. Une calligraphie danse l'écriture, le signe. Elle redéfinit le linéaire. Le corps calligraphique est celui où se met en scène une cartographie du désir, de la sensation, de l'intensité et des flux. Il donne à voir, se donne à voir, ne représente pas mais présente.

Aujourd'hui, au Maghreb, certains partis islamistes menacent la culture maghrébine au nom d'une identité islamo-arabe. Le corps dansant maghrébin se veut artiste, créateur, résistant et multiple. Tunisien, marocain, algérien ou libyen, berbère ou juif, il est avant tout un corps de danse. À l'heure où le Maghreb (avec l'ensemble du monde arabe) vit des révolutions, des insurrections et des changements politiques majeurs, le moment est venu sans doute de repenser l'entrée de ses arts propres dans une nouvelle période de l'histoire, de penser un art contemporain maghrébin.

\section{Mariem Guellouz}

